\title{
Staphylococcus Aureus carriage and long-term Rituximab treatment for Granulomatosis with polyangiitis
}

Emilio Besada, Wenche Koldingsnes, Johannes C Nossent

Objective: Chronic nasal carriage of Staphylococcus aureus (SA) increases the risk of relapse while Rituximab (RTX) is an effective agent for inducing and maintaining remission in patients with Granulomatosis with polyangiitis (GPA). We investigated whether B cell depletion and hypogammaglobulinemia that occur during RTX treatment increase the risk of chronic SA nasal carriage and subsequent disease flares, in GPA patients on long-term RTX maintenance therapy. Methods: Retrospective cohort study from a disease registry involving 29 GPA patients receiving RTX maintenance (median RTX dose of $9 \mathrm{~g}$ ) during a median period of 49 months. Nasal swabs were collected prior and during RTX for a median of 3 and 9 swabs respectively. Persistent SA nasal carriage was defined with the presence of SA in more than $75 \%$ of nasal swabs. Results: SA nasal carriage did not change during RTX ( $p=0.297)$. However, the rate of positive nasal swabs in GPA patients with transient SA nasal carriage during RTX maintenance increased from 0 prior RTX to 0.42 during RTX ( $p=0.017)$. Persistent SA nasal carriage did not increase the risk of relapses $(p=0.844)$, of hypogammaglobulinemia $(p=0.122)$ and of severe infections $(p=0.144)$, but reduced the risk of chronic infections $(p=0.044)$. Change in SA carriage status during RTX did not influence the risk of relapses $(p=0.756)$,

hypogammaglobulinamia $(p=0.474)$ and infections, either severe $(p=0.913)$ or chronic $(p=0.121)$. Conclusion: Long-term RTX maintenance therapy in GPA patients did not significantly influence SA nasal carriage status. Persistent SA carriage during long-term RTX treatment did not seem to increase the risk of relapses, but seemed to decrease the risk of hypogammaglobulinemia associated chronic infections. 
2 Staphylococcus Aureus carriage and long-term Rituximab treatment for Granulomatosis with

3 polyangiitis

4

5

6 Emilio Besada ${ }^{(1)}$, Wenche Koldingsnes ${ }^{(2)}$ and Johannes C Nossent ${ }^{(1,3)}$

7

14 Corresponding author:

15 Emilio Besada

16 Bone and joint research group, Department of Clinical Medicine, Faculty of Health Sciences,

17 UiT The Arctic University of Norway, 9037 Tromsø, Norway

18 Telephone: +4777627294

19 Fax: +4777627258

20 e-mail: emilio.besada@uit.no

21

Keywords: infection, vasculitis, maintenance, antibiotic prophylaxis, nasal barrier. 


\section{ABSTRACT \\ Objective:}

Chronic nasal carriage of Staphylococcus aureus (SA) increases the risk of relapse while Rituximab (RTX) is an effective agent for inducing and maintaining remission in patients with Granulomatosis with polyangiitis (GPA). We investigated whether B cell depletion and hypogammaglobulinemia that occur during RTX treatment increase the risk of chronic SA nasal carriage and subsequent disease flares, in GPA patients on long-term RTX maintenance therapy. Methods:

Retrospective cohort study from a disease registry involving 29 GPA patients receiving RTX maintenance (median RTX dose of $9 \mathrm{~g}$ ) during a median period of 49 months. Nasal swabs were collected prior and during RTX for a median of 3 and 9 swabs respectively. Persistent SA nasal carriage was defined with the presence of SA in more than $75 \%$ of nasal swabs.

Results:

SA nasal carriage did not change during RTX ( $p=0.297)$. However, the rate of positive nasal swabs in GPA patients with transient SA nasal carriage during RTX maintenance increased from 0 prior RTX to 0.42 during $\mathrm{RTX}(\mathrm{p}=0.017)$.

Persistent SA nasal carriage did not increase the risk of relapses $(\mathrm{p}=0.844)$, of hypogammaglobulinemia $(\mathrm{p}=0.122)$ and of severe infections $(\mathrm{p}=0.144)$, but reduced the risk of chronic infections $(\mathrm{p}=0.044)$. Change in SA carriage status during RTX did not influence the risk of relapses $(\mathrm{p}=0.756)$, hypogammaglobulinamia $(\mathrm{p}=0.474)$ and infections, either severe $(\mathrm{p}=0.913)$ or chronic $(\mathrm{p}=0.121)$.

Conclusion: 
49 Long-term RTX maintenance therapy in GPA patients did not significantly influence SA nasal

50 carriage status. Persistent SA carriage during long-term RTX treatment did not seem to increase

51 the risk of relapses, but seemed to decrease the risk of hypogammaglobulinemia associated

52 chronic infections. 


\section{INTRODUCTION}

Granulomatosis with polyangiitis (GPA) is a clinicopathologic variant of antineutrophil cytoplasmic antibodies (ANCA)-associated vasculitis (AAV). It is characterized by a necrotizing granulomatous inflammation usually involving the upper and lower respiratory tract, a necrotizing vasculitis affecting predominantly small to medium vessels and commonly a necrotizing glomerulonephritis, often in the presence of Proteinase 3 (PR3)-ANCA [1]. The aetiology of GPA is unknown; but infections and in particular nasal carriage of Staphylococcus aureus (SA) have been implicated [2]. SA nasal carriage may trigger GPA through staphylococcal superantigens, molecular mimicry, increased Toll-like receptor signalling in leukocytes and the induction of neutrophil extracellular traps (NETs) [2]. SA interacts with endothelial, T and B cells creating an inflammatory milieu and activating neutrophils locally responsible of damage by release of proteolytic enzymes and reactive oxygen species [3]. SA can evade innate immunity by resisting phagocytosis and can survive inside neutrophils [4]. SA can also evade induced immunity since SA can deplete potential antibody-secreting B cells and inhibit proliferation of antigen-specific T cells [4]. GPA patients have an abnormal epithelial nasal barrier with an altered basal cytokine expression and a reduced secretion of interleukin- 8 upon SA stimulation that could facilitate nasal carriage [5].

SA permanently colonises the anterior nares of $20 \%$ of the population while transient colonisation occurs in another $60 \%$ [6]. In one study, GPA patients have a slightly increased rate of SA nasal carriage (72\%), compared to Rheumatoid Arthritis patients (46\%) and hospital staff members (58\%) [7]. The risk of disease relapses in GPA increases with SA nasal carriage [7] and is 7 times higher with chronic SA nasal carriage [8]. Antibiotic prophylaxis with Trimethoprim-sulfamethoxazole (TMP-SMX) can decrease the risk of relapse by $60 \%$ and of 
77 infections in GPA $[9,10]$, although the basis for this is unclear since TMP-SMX antibiotic

78 prophylaxis does not eliminate SA nasal carriage in GPA [5,11].

79 While immunosuppressive drugs (ID) are the mainstay of therapy in GPA, B cell depleting

80 therapy with rituximab (RTX) has proven very effective in inducing $[12,13]$ and maintaining

81 remission in GPA [14-16]. However, side effects of B cell depletion over time such as the

82 reduction in all immunoglobulins (Ig) class levels [16] could potentially increase the risk of SA

83 carriage.

84 Our study investigated if long-term RTX maintenance treatment increases the risk of SA carriage

85 in GPA patients and if SA carriage promoted relapses, infections and/or

86 hypogammaglobulinemia. 
PATIENTS AND METHODS

89 Since 2001 our vasculitis disease registry (Nordnorsk Vaskulittregister) has collected information on disease presentation and course from patients with an established diagnosis of

91 primary vasculitis followed at the University Hospital of North Norway. All patients gave informed written consent at registry inclusion according to the declaration of Helsinki. All patients satisfied the American College of Rheumatology 1990 classification and/or Chapel Hill consensus conference criteria [17-18]. Twenty-nine GPA patients from that registry who received long-term RTX treatment with a RTX cumulative dose of $5 \mathrm{~g}$ or more before September 2011 were included in the study. $86 \%$ received RTX for relapse and $14 \%$ for new disease. RTX treatment was initiated as two 1-gram infusions 2 weeks apart with co-administration of methylprednisolone $125 \mathrm{mg}$, paracetamol $1000 \mathrm{mg}$ and either cetirizine $10 \mathrm{mg}$ or polaramine 4 mg (RA protocol). Due to the observed RTX efficacy and the relapsing nature of GPA, RTX was then re-administered pre-emptively either as $2 \mathrm{~g}$ infusion ( $1 \mathrm{~g}$ twice during a fortnight) annually or as $1 \mathrm{~g}$ infusion biannually ( $1 \mathrm{~g}$ every 6 months). RTX was added to other ID (other than

102 prednisolone) in 27 patients (93\%), while $2(7 \%)$ received RTX in monotherapy. The timing and 103 pace of ID discontinuation was at the discretion of the treating physician, whereas the oral daily 104 prednisolone dose (ODPD) was tapered and discontinued in a controlled manner. Antibiotic prophylaxis against relapse with TMP-SMX (dose 320mg of TMP and 1600mg of

107 SMX daily) was not blinded and was at the physician's discretion. Ciprofloxacin (dose ranging 108 from 500 to $1000 \mathrm{mg}$ daily) was used in case of sulfamide allergy, poor TMP-SMX tolerance or concomitant methotrexate use. Compliance of antibiotic prophylaxis was assessed during 
110 patients' visits. We defined antibiotic prophylaxis as the use of antibiotic prophylaxis for more

111 than $50 \%$ of the time during long-term RTX maintenance.

112 Nasal swabs were performed biannually in one anterior naris by firmly rotating the sterile cotton-

113 swab. Similar to the study of Stegeman el al, we defined patients with positive SA nasal carriage

114 as either transient or persistent if SA was present in respectively less than $75 \%$ and more than 75

$115 \%$ of all the nasal swabs [8]. We defined patients as non-SA carriers when none of the nasal

116 swabs grew SA.

117 Fixed set of blood tests (ANCA levels, quantification of immunoglobulin classes and flow

118 cytometric immunophenotyping of lymphocytes) were performed prior RTX initiation and

119 before each new re-treatment until either $30^{\text {th }}$ September 2011 (closing date of this study) or

120 shortly before the administration of intravenous immunoglobulins (IVIG) due to

121 hypogammaglobulinemia and/or infections. CD4 cell counts and serum total Ig levels taken after

122 the administration of $2 \mathrm{~g}$ of RTX and at last visit were included in the study.

123

124 Relapses were defined by the recurrence, worsening or first appearance of one or more BVAS

125 items attributable to GPA after remission. Hypogammaglobulinemia was defined as serum total

$126 \operatorname{Ig}<6 \mathrm{~g} / \mathrm{L}$. Severe infections were defined as if hospitalisation and intravenous antibiotic

127 treatment were necessary, while chronic infections were defined as symptomatic infections

128 mostly localised in the ear-nose-throat (ENT) and respiratory tract, lasting 3 months or more and

129 requiring several antibiotic courses.

130

131 Data were analysed with SPSS version 20.0 (SPSS Ltd, Chicago IL, USA). Descriptive statistics

132 were used to describe SA nasal carriage during RTX maintenance. Continuous variables were 
133 expressed in median and range; categorical variables were expressed in number and percentage.

134 Related-samples Wilcoxon signed rank test was used to test for difference between rates of

135 positive SA nasal swabs prior RTX and during maintenance. Chi-square and Kruskal Wallis tests

136 were used appropriately for subgroups analysis concerning SA nasal carriage status. SA nasal

137 carriage, change in SA carriage status and antibiotic prophylaxis during RTX maintenance were

138 analysed by Kaplan-Meier survival curves as risk factors of relapse, infections and RTX

139 discontinuation due to hypogammaglobulinemia. P-values $<0.05$ were considered significant. 
141

142

143 Twenty-nine GPA patients (52\% men) with a median age of 50 years (19-75) received RTX. 90

$144 \%$ of the patients were ANCA positive at diagnosis: $86 \%$ PR3-ANCA and $3 \%$ MPO-ANCA

145 positive. $59 \%$ had renal involvement; $66 \%$ had pulmonary involvement and $62 \%$ had orbital-

146 subglottic involvement. At RTX initiation, their disease duration was 57 (22-70) months and

147 they had received a cumulative dose of 17 (0-250) g cyclophosphamide.

148 GPA patients received a cumulative RTX dose of $9 \mathrm{~g}(5-13)$ for remission induction and

149 maintenance during 49 (19-88) months. Twelve patients (41\%) received the $1 \mathrm{~g}$ biannually

150 regimen, $6(21 \%)$ received the $2 \mathrm{~g}$ annually regimen and $11(38 \%)$ alternated between regimens.

151 RTX was added to other ID (other than prednisolone) in 27 patients (93\%), which was

152 continued during 24 months (1-54) after RTX initiation. ODPD decreased from 23 (0-60) at

153 baseline to $5(0-15) \mathrm{mg}$ at last visit. Six patients (21\%) discontinued prednisolone 23 months (7-

$15455)$ after RTX initiation.

155

156

157

158

160

161

162

163

Nasal carriage during RTX maintenance

Three (1-8) nasal swabs were analysed before RTX and 9 (2-22) during RTX maintenance. The number of nasal swabs did not differ between the different SA carriage groups before and during RTX (respectively $\mathrm{p}=0.411$ and $\mathrm{p}=0.437$ ).

Before RTX initiation, 13 patients (52\%) were non-SA carriers, but 2 patients had positive swabs for other bacteria (Table 1). Seven patients (28\%) had transient SA nasal carriage and 5 (20\%) had persistent carriage before RTX. During RTX maintenance, 9 patients $(31 \%)$ were 
164 non-SA carriers, while $12(41 \%)$ had transient carriage and $8(28 \%)$ had persistent nasal

165 carriage. Ten (40\%) patients did not change SA carriage status during RTX maintenance, while

$16611(44 \%)$ increased nasal SA carriage status and $4(16 \%)$ decreased during RTX maintenance.

167 The frequency of SA nasal carriage did not differ significantly before and during RTX ( $\mathrm{p}=0.297)$

168 (Table 1). However, the rate of positive nasal swabs in GPA patients with transient SA nasal

169 carriage during RTX maintenance $(n=11)$ increased from 0 prior RTX to 0.42 during RTX

$170 \quad(\mathrm{p}=0.017$ after square root transformation).

171 The nasal swabs identified several other bacteria at initiation and during RTX maintenance

172 (Table 1). Of interest, 7 (24\%) and $4(14 \%)$ GPA patients had respectively Streptococcus

173 pneumoniae and Haemophilus influenzae at least once in their nasal swabs during RTX

174 maintenance. S. pneumoniae and SA were often identified together at last visit, whereas H.

175 influenzae was identified in 2 out of 8 patients who discontinued RTX due to

176 hypogammaglobulinemia at the time of its discontinuation.

177

178

$179 \quad$ SA nasal carriage

180 GPA patient characteristics differed in relation to SA nasal carriage. SA non-carriers had less

181 lung involvement $(\mathrm{p}=0.047)$ and seemed to receive higher ODPD at baseline $(\mathrm{p}=0.141)$

182 compared with patients with either transient or persistent nasal carriage (Table 2). On the other

183 hand, patients with persistent SA nasal carriage had received $55 \mathrm{~g}$ of CYC compared with $13 \mathrm{~g}$ in

184 the other 2 subgroups ( $\mathrm{p}=0.163$ ). The use of other ID during RTX treatment was similar within

185 the different SA nasal carriage subgroups (Table 2). 
188 During RTX maintenance, 9 relapses occurred in 8 patients $(28 \%)$ and 2 thirds of the relapses

189 involved the respiratory tract and ENT. Three patients (38\%) who relapsed had persistent SA

190 nasal carriage (Table 2). The lone patient who relapsed twice did not carry SA. There was no

191 association between SA nasal carriage and either overall frequency of relapses $(p=0.243)$ (Table

192 2) or time-adjusted risk of relapse $(\mathrm{p}=0.844)$ (Figure 1A). There was no association between

193 change of SA nasal carriage status during RTX and time-adjusted risk of relapse $(p=0.756)$

194 (Figure 2A).

195

196

$\underline{\text { Risk of infections }}$

197 During RTX maintenance, severe and chronic infections developed in 7 and 9 patients

198 respectively.

199 Seven patients developed severe infections, but there was no association between SA carriage

200 and the frequency $(\mathrm{p}=0.213)$ (Table 2$)$ or time-adjusted risk $(\mathrm{p}=0.144)$ (Figure 1B) of severe

201 infections. There was no association between change of SA nasal carriage status during RTX and

202 time-adjusted risk of relapse ( $\mathrm{p}=0.913)$ (Figure 2B).

203 Nine patients had chronic infections; however none of these patients had persistent SA nasal

204 carriage $(\mathrm{p}=0.082)$ (Table 2). Patients with persistent SA nasal carriage and patients with an

205 increase of SA nasal carriage status seemed to have a lower time-adjusted risk of developing 206 chronic infections (respectively $\mathrm{p}=0.044$ and $\mathrm{p}=0.125$ ) (Figures $1 \mathrm{C}$ and $2 \mathrm{C}$ ). Of interest, CD4

207 cell count seemed to increase in persistent and transient carriers, but seemed to decrease in non208 carriers following the first $2 \mathrm{~g}$ of RTX $(\mathrm{p}=0.071)$ (Table 2). 
211 Eight patients discontinued RTX due to hypogammaglobulinemia with a level of serum total Ig

212 of $4.7(3.5-5.5) \mathrm{g} / \mathrm{L}$. Four patients $(50 \%)$ had persistent or transient SA nasal carriage, while 4

213 patients (50\%) did not carry SA (Table 2). Patients with transient SA nasal carriage had a

214 tendency to lower time-adjusted risk of discontinuing RTX due to hypogammaglobulinemia,

215 although this was not statistically significant ( $\mathrm{p}=0.122$ ) (Figure 1D). Change in SA nasal carriage

216 status during RTX did not influence the risk of discontinuing RTX due to

217 hypogammaglobulinemia $(\mathrm{p}=0.474)$ (Figure 2D).

218 Non-SA carriers had a tendency to more profound decline of serum total Ig both after the first 2

$219 \mathrm{~g}$ of RTX $(\mathrm{p}=0.079)$ and during RTX maintenance $(\mathrm{p}=0.063)$ compared with patients with either

220 transient or persistent SA carriage (Table 2).

221

222

223

TMP-SMX and ciprofloxacin during RTX

224 Before RTX initiation, most patients (90 \%) had received antibiotic prophylaxis with either

225 TMP-SMX or ciprofloxacin at some point during the course of their disease. However, only 16

226 patients (55\%) received TMP-SMX antibiotic prophylaxis during RTX maintenance and just 6

227 (21\%) used TMP-SMX for more than $50 \%$ of the time. Similarly, 10 patients $(35 \%)$ received

228 ciprofloxacin during RTX maintenance and 7 (24\%) used it for more than $50 \%$ of the time.

229 Two patients (7 \%) switched from ciprofloxacin to TMP-SMX, while no patients switched from

230 TMP-SMX to ciprofloxacin during RTX maintenance. The first patient switched when

231 methotrexate was discontinued. The other switched with the intention of preventing relapse when

232 mycophenolate mofetil was discontinued (even though the nasal swab was negative). ODPD, 
233 CYC cumulative dose and concomitant use of ID during RTX maintenance did not differ

234 between patients receiving either TMP-SMX or ciprofloxacin and patients not receiving 235 antibiotics.

236

237 During RTX treatment, none of the six GPA patients who used TMP-SMX for more than $50 \%$ 238 of the time had persistent SA nasal carriage and three (50\%) were non-SA carriers. In the 239 ciprofloxacin group, $2(29 \%)$ had persistent SA nasal carriage and $2(29 \%)$ were non-SA 240 carriers. Patients who used TMP-SMX for more than $50 \%$ of the time during RTX maintenance 241 had an increased risk to hypogammaglobulinemia leading to RTX discontinuation $(\mathrm{p}=0.046)$

242 (Figure 3A). On the other hand, none of the patients using ciprofloxacin for more than $50 \%$ of

243 the time during RTX maintenance seemed to discontinue RTX due to hypogammaglobulinemia $244(\mathrm{p}=0.065)$ (Figure 3B). There were no significant differences for the risk of severe infections, 245 chronic infections and relapses risks in patients on or off antibiotic prophylaxis (with either 246 TMP-SMX or ciprofloxacin) (data not shown). 
DISCUSSION

249 The rate of persistent SA nasal carriage did not change significantly in GPA patients during

250 long-term RTX treatment, even though SA nasal carriage seemed to increase in patients with

251 transient carriage. Persistent SA nasal carriage was not a risk factor for disease relapses, but was

252 associated with a lower frequency of chronic infections. On the other hand, non-SA carriers

253 seemed to have lower CD4 counts and a steeper decline of total Ig after 2 $g$ of RTX and were

254 more prone to infections and to discontinue RTX due to hypogammaglobulinemia.

256 Contrary to the seminal study of Stegeman et al [8], persistent SA nasal carriage did not increase

257 the risk of relapse in GPA patients whilst on treatment with RTX. This may be due to the lower

258 overall frequency of SA carriers in this cohort since $63 \%$ were persistent SA nasal carriers in the

259 Stegeman study [8], but only $20 \%$ and $28 \%$ persistently carried SA in our cohort before and

260 during RTX maintenance respectively. Nasal swabs procedure and inclusion as well as patient

261 selection bias could explain the difference. Nasal swabs were performed unilaterally in our study

262 and bilaterally in the study of Stegeman [8]. Nasal swabs of GPA patients receiving prolonged

263 antibiotics defined as more than 6 weeks were excluded in the study of Stegeman [8], whereas all

264 nasal swabs in our study were included with or without concomitant antibiotics. Also while

265 Stegeman et al enrolled all consecutive patients diagnosed with GPA over a 3.5 years period [8],

266 our study included consecutive GPA patients receiving long-term RTX maintenance, who had

267 been mostly refractory to conventional therapy.

268

269 The frequency of severe infections was equal in persistent and non-SA carriers, but the risk of

270 chronic infections during RTX treatment seemed lower in persistent SA carriers and in patients 
271 with increased SA nasal carriage status. In healthy individuals, persistent SA nasal carriers have 272 a higher risk of infection [19] than transient and non-carriers who eradicate SA faster due to

273 stronger anti-staphylococcal antibodies response [20]. In our study, non-SA carriers

274 paradoxically seemed to have a more profound decline of total serum Ig and CD4 cell count

275 already after $2 \mathrm{~g}$ of RTX, indicating an early effect of RTX on the ability to mount an effective

276 immune response in non-SA carriers.

277 In healthy individuals, SA carriage also influences the nasal microbiome and inhibits

278 colonisation from other bacteria [19]. Consequently, persistent SA nasal carriage in GPA patients

279 receiving long-term RTX could prevent colonisation from other bacteria and chronic infections

280 due to the continuing interplay between SA and the immune system.

281

282 In our selected cohort of closely followed GPA patients, antibiotic prophylaxis was neither 283 universal nor continuous and this reflects the uncertainties regarding this issue [7]. TMP-SMX 284 use during RTX maintenance seemed to increase the risk of hypogammaglobulinemia and RTX 285 discontinuation and to decrease persistent SA nasal carriage, compared with ciprofloxacin. While 286 TMP-SMX therapy eliminates neither SA nasal carriage nor endonasal activity in GPA [7], the 287 combination of TMP-SMX and RTX could provoke changes in the nasal epithelial barrier and its 288 microbiome that might be relevant for the course of the disease. Moreover, TMP-SMX could 289 exert an additional anti-inflammatory effect by interfering with the production of reactive oxygen 290 species in neutrophils [21]. As TMP-SMX decreases the risk of relapse [9], it could, in addition, 291 increase the risk to discontinue RTX due to hypogammaglobulinemia. 292 
293 Our retrospective study is limited by its sample size with the low number of events making 294 subgroups analysis prone to type II errors of not detecting significant differences. Patients'

295 selection with multiple relapses and confounding factors, such as high CYC cumulative dose,

296 could have biased our results and limit their applicability. Despite this, our study is the first that

297 highlights a possible association between SA carriage status, infections and

298 hypogammaglobulinemia during RTX therapy in GPA patients.

299

300 In conclusion, SA nasal carriage in GPA patients did not change significantly during long-term

301 RTX maintenance. Non-SA carriers were as likely to have disease flares but were more prone to

302 infections and to discontinue RTX due to hypogammaglobulinemia independently of the CYC

303 cumulative dose. These results suggest that long-term RTX treatment is better tolerated in GPA 304 patients with SA nasal carriage.

305

306 AFFILIATIONS:

307 W Koldingsnes has received consulting fees, speaking fees and travel grants from Hoffmann-La 308 Roche.

309

310 FUNDING:

311 The authors declare there was no funding for this work. 


\section{REFERENCES}

314 1. Jennette JC, Falk RJ, Bacon PA, Basu N, Cid MC, Ferrario F, Flores-Suarez LF, Gross

315

316

317 WL, Guillevin L, Hagen EC, Hoffman GS, Jayne DR, Kallenberg CG, Lamprecht P, Langford CA, Luqmani RA, Mahr AD, Matteson EL, Merkel PA, Ozen S, Pusey CD, Rasmussen N, Rees AJ, Scott DG, Specks U, Stone JH, Takahashi K, Watts RA. 2012 revised International Chapel Hill Consensus Conference Nomenclature of Vasculitides. Arthritis Rheum 2013;65:1-11

2. Tadema H, Heeringa P, Kallenberg CG. Bacterial infections in Wegener's Granulomatosis: mechanisms potentially involved in autoimmune pathogenesis. Curr Opin Rheumatol 2011;23:366-71

3. Popa ER, Tervaert JW. The relation between Staphylococcus aureus and Wegener's granulomatosis: current knowledge and future directions. Intern Med 2003:42:771-80

4. Foster TJ. Immune evasion by staphylococci. Nat Rev Microbiol 2005;3:948-58.

5. Wohlers J, Breucker K, Podschun R, Hedderich J, Lamprecht P, Ambrosch P, Laudien M. Aberrant cytokine pattern of the nasal mucosa in granulomatosis with polyangiitis. Arthritis Res Ther 2012;14:R203

6. Peacock SJ, de Silva I, Lowy FD. What determines nasal carriage of Staphylococcus aureus? Trends Microbiol 2001;9:605-10

7. Laudien M, Gadola SD, Podschun R, Hedderich J, Paulsen J, Reinhold-Keller E, Csernok E, Ambrosch P, Hellmich B, Moosig F, Gross WL, Sahly H, Lamprecht P. Nasal carriage of Staphylococcus aureus and endonasal activity in Wegener's granulomatosis as compared to rheumatoid arthritis and chronic rhinosinusitis with nasal polyps. Clin Exp Rheumatol 2010;28(Suppl. 57):S51-S55 
8. Stegeman CA, Tervaert JW, Sluiter WJ, Manson WL, de Jong PE, Kallenberg CG. Association of chronic nasal carriage of Staphylococcus aureus and higher relapse rates in Wegener Granulomatosis. Ann Intern Med 1994;120:12-7

9. Stegeman CA, Tervaert JW, de Jong PE, Kallenberg CG. Trimethoprim-

10. Zycinska K, Wardyn KA, Zielonka TM, Krupa R, Lukas W. Co-trimoxazole and sulfamethoxazole (co-trimoxazole) for the prevention of relapses of Wegener's Granulomatosis. N Engl J Med 1996;335:16-20

12. Jones RB, Tevaert JW, Hauser T, Luqmani R, Morgan MD, Peh CA, Savage CO, Segelmark M, Tesar V, van Paassen P, Walsh D, Walsh M, Westman K, Jayne DR; European Vasculitis Study Group. Rituximab versus cyclophosphamide in ANCAassociated renal vasculitis. N Engl J Med 2010;363:211-20

13. Stone JH, Merkel PA, Spiera R, Seo P, Langford CA, Hoffman GS, Kallenberg CG, St Clair EW, Turkiewicz A, Tchao NK, Webber L, Ding L, Sejismundo LP, Mieras K, Weitzenkamp D, Ikle D, Seyfert-Margolis V, Mueller M, Brunetta P, Allen NB, Fervenza FC, Geetha D, Keogh KA, Kissin EY, Monach PA, Peikert T, Stegeman C, Ytterberg SR, 
Specks U; RAVE-ITN Research Group. Rituximab versus cyclophosphamide for ANCAassociated vasculitis. N Engl J Med 2010;363:221-32

14. Smith RM, Jones RB, Guerry MJ, Laurino S, Catapano F, Chaudhry A, Smith KG, Jayne Arthritis Rheum 2012;64:3760-69

15. Cartin-Ceba R, Golbin JM, Keogh KA, Peikert T, Sánchez-Menéndez M, Ytterberg SR, Fervenza FC, Specks U. Rituximab for remission induction and maintenance in refractory granulomatosis with polyangiitis (Wegener's): ten-year experience at a single center. Arthritis Rheum 2012;64:3770-8

16. Besada E, Koldingsnes W, Nossent JC. Long-term efficacy and safety of pre-emptive maintenance therapy with rituximab in granulomatosis with polyangiitis: results from a single centre. Rheumatology 2013;52:2041-7

17. Jennette JC, Falk RJ, Andrassy K, Bacon PA, Churg J, Gross WL, Hagen EC, Hoffman GS, Hunder GG, Kallenberg CG, Mccluskey RT, Sinico RA, Rees AJ, Van Es LA, Waldherr, Wiik A. Nomenclature of systemic vasculitides; proposal of an International Consensus Conference. Arthritis Rheum 1994;37:187-92

18. Leavitt RY, Fauci AS, Bloch DA, Michel BA, Hunder GG, Arend WP, Calabrese LH, Fries JF, Lie JT, Lightfoot RW Jr, Masi AT, McShane DJ, Mills JA, Stevens MB, Wallace SL and Zvaifler NJ. The American College of Rheumatology 1990 criteria for the classification of Wegener's granulomatosis. Arthritis Rheum 1990;33:1101-7

19. Johannessen M, Sollid JE, Hanssen AM. Host and microbe determinants that may influence the success of S. aureus colonization. Front Cell Infect Microbiol 2012;2:56. doi: $10.3389 /$ fcimb.2012.00056 
381 20. van Belkum A, Verkaik NJ, de Vogel CP, Boelens HA, Verveer J, Nouwen JL, Verbrugh

382 HA, Wertheim HF. Reclassification of Staphylococcus aureus nasal carriage types. J

$383 \quad$ Infect Dis 2009;199:1820-6

384 21. Roberts DE, Curd JD. Sulfonamides as antiinflammatory agents in the treatment of

$385 \quad$ Wegener's granulomatosis. Arthritis Rheum 1990;33:1590-3

386 


\section{Table $\mathbf{1}_{\text {(on next page) }}$}

Staphylococcus aureus and other bacteria nasal carriage before and during rituximab maintenance in granulomatosis with polyangiitis patients

RTX: rituximab Results are expressed in absolute numbers (percentage) and significance is determined by Chi-squared test for association. Footnotes: a. No nasal swabs results were recorded in 4 patients. b. One patient had Pseudomonas aeruginosa prior to RTX. c. One patient had Pseudomonas aeruginosa in 2 nasal swabs and one patient had Neisseria species found in one nasal swab. [b] 


\section{Table 1}

3

4 Staphylococcus aureus and other bacteria nasal carriage before and during rituximab maintenance in

5 granulomatosis with polyangiitis patients

6

\section{Prior RTX During RTX p}

(25) ${ }^{\mathrm{a}}$

(29)

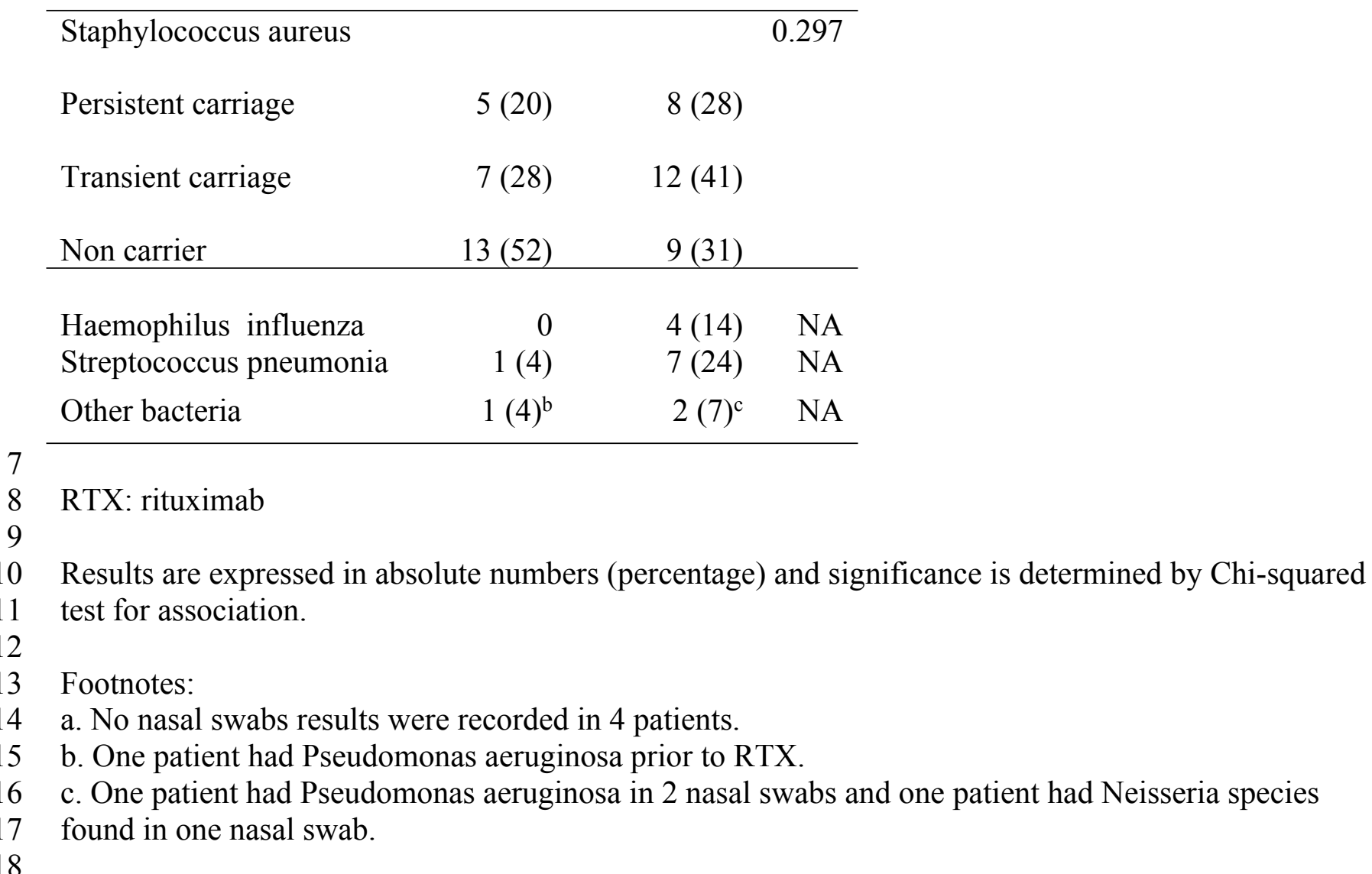




\section{Table 2 (on next page)}

Granulomatosis with polyangiitis patients' characteristics determined by Staphylococcus aureus nasal carriage during long-term rituximab maintenance.

AZA: azathioprine; BVAS: Birmingham vasculitis activity score; CD: cluster of differentiation;

CYC: cyclophosphamide; Ig: immunoglobulins; MMF: mycophenolate mofetil; MTX:

methotrexate; PR3-ANCA: proteinase 3 antineutrophil cytoplasmic antibodies; RTX:

rituximab; SA: Staphylococcus aureus. TMP-SMX: trimethoprim-sulfamethoxazole Results are expressed in medians for continuous variables and in absolute numbers (percentages) for categorical variables. Difference is determined by Kruskal Wallis Test for continuous variables and Chi-square test for categorical variables. 
2 Table 2

3 Granulomatosis with polyangiitis patients' characteristics determined by Staphylococcus aureus nasal

4 carriage during long-term rituximab maintenance.

5

\begin{tabular}{|c|c|c|c|c|}
\hline & No carriage & $\begin{array}{l}\text { Transient } \\
\text { carriage }\end{array}$ & $\begin{array}{c}\text { Persistent } \\
\text { carriage }\end{array}$ & $\begin{array}{c}\mathrm{p}- \\
\text { value }\end{array}$ \\
\hline & 9 patients & 12 patients & 8 patients & \\
\hline Male & $5(56)$ & $5(42)$ & $5(62)$ & 0.634 \\
\hline Age at baseline (y) & 54 & 43 & 44 & 0.375 \\
\hline Kidney involvement & $5(56)$ & $6(50)$ & $6(75)$ & 0.525 \\
\hline Lung involvement & $3(33)$ & $10(83)$ & $6(75)$ & 0.047 \\
\hline Orbital-subglottic involvement & $4(44)$ & $9(75)$ & $5(63)$ & 0.361 \\
\hline PR3-ANCA & $8(89)$ & $10(83)$ & $7(88)$ & 0.414 \\
\hline BVAS at baseline & 11 & 10 & 10 & 0.382 \\
\hline $\begin{array}{l}\text { RTX maintenance duration } \\
\text { (w) }\end{array}$ & 173 & 182 & 170 & 0.305 \\
\hline CYC cumulative dose (g) & 13 & 13 & 55 & 0.163 \\
\hline RTX cumulative dose (g) & 8 & 9.5 & 8 & 0.468 \\
\hline 1g biannually regimen & $6(67)$ & $3(25)$ & $3(38)$ & 0.153 \\
\hline MTX use during RTX & $3(33)$ & $4(33)$ & $4(50)$ & 0.711 \\
\hline AZA use during RTX & $3(33)$ & $3(25)$ & $2(25)$ & 0.898 \\
\hline MMF use during RTX & $1(11)$ & $5(42)$ & $2(25)$ & 0.295 \\
\hline CYC use during RTX & $3(33)$ & $3(25)$ & $3(38)$ & 0.891 \\
\hline Total Ig at baseline $(\mathrm{g} / \mathrm{L})$ & 11 & 8.5 & 11 & 0.278 \\
\hline Total Ig after RTX $2 g(g / L)$ & 8.1 & 7.5 & 9.4 & 0.730 \\
\hline CD4 at baseline $\left(\times 10^{9} / \mathrm{L}\right)$ & 0.39 & 0.40 & 0.27 & 0.538 \\
\hline CD4 after RTX $2 \mathrm{~g}\left(\times 10^{9} / \mathrm{L}\right)$ & 0.28 & 0.45 & 0.39 & 0.071 \\
\hline $\begin{array}{l}\text { Total Ig decline after RTX } 2 \mathrm{~g} \\
(\mathrm{~g} / \mathrm{L})\end{array}$ & 3.5 & 1.4 & 1.9 & 0.079 \\
\hline $\begin{array}{l}\text { Total Ig overall decline during } \\
\text { RTX }\end{array}$ & 5.1 & 2.5 & 3.4 & 0.063 \\
\hline TMP-SMX during RTX & $3(33)$ & $3(25)$ & 0 & 0.212 \\
\hline Ciprofloxacin during RTX & $2(22)$ & $3(25)$ & $2(25)$ & 0.987 \\
\hline Severe infections & $3(33)$ & $1(8)$ & $3(38)$ & 0.243 \\
\hline Chronic infections & $4(44)$ & $5(42)$ & 0 & 0.082 \\
\hline Relapses & $2(22)$ & $3(25)$ & $3(38)$ & 0.754 \\
\hline $\begin{array}{l}\text { RTX discontinuation due to } \\
\text { hypogammaglobulinemia }\end{array}$ & $4(44)$ & $2(17)$ & $2(25)$ & 0.364 \\
\hline
\end{tabular}

6

7 AZA: azathioprine; BVAS: Birmingham vasculitis activity score; CD: cluster of differentiation; CYC:

8 cyclophosphamide; Ig: immunoglobulins; MMF: mycophenolate mofetil; MTX: methotrexate; PR3- 
9 ANCA: proteinase 3 antineutrophil cytoplasmic antibodies; RTX: rituximab; SA: Staphylococcus 10 aureus. TMP-SMX: trimethoprim-sulfamethoxazole

11

12 Results are expressed in medians for continuous variables and in absolute numbers (percentages) for 13 categorical variables. Difference is determined by Kruskal Wallis Test for continuous variables and 14 Chi-square test for categorical variables.

15

16 
Figure 1 (on next page)

Kaplan-Meier analysis of the probability of relapse (A), severe (B) and chronic (C) infections and discontinuation due to hypogammaglobulinemia (D) according to Staphylococcus aureus nasal carriage status

Figure 1 
A Log rank $p=0.844$

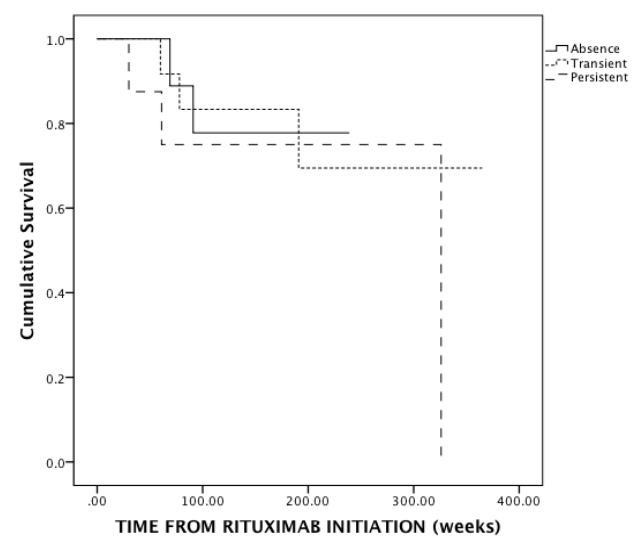

\begin{tabular}{l|r|r|r|r} 
Week & 0 & 100 & 200 & 300 \\
\hline $\mathrm{N}$ & 29 & 22 & 10 & 2
\end{tabular}

C Log rank $\mathrm{p}=\mathbf{0 . 0 4 4}$

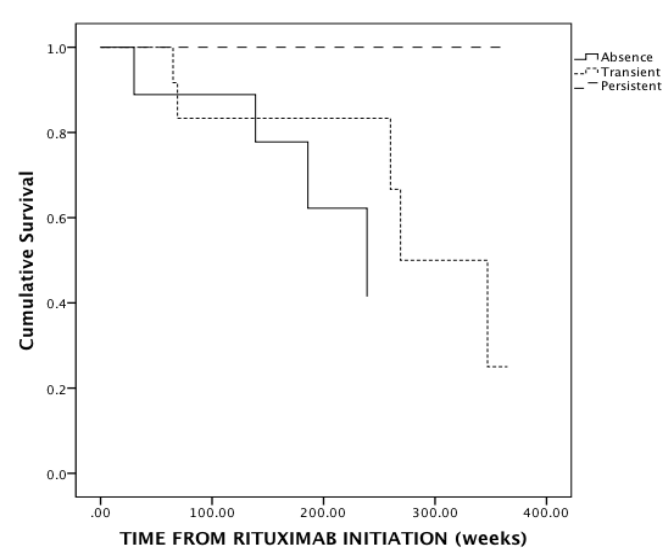

B Log rank $p=0.144$

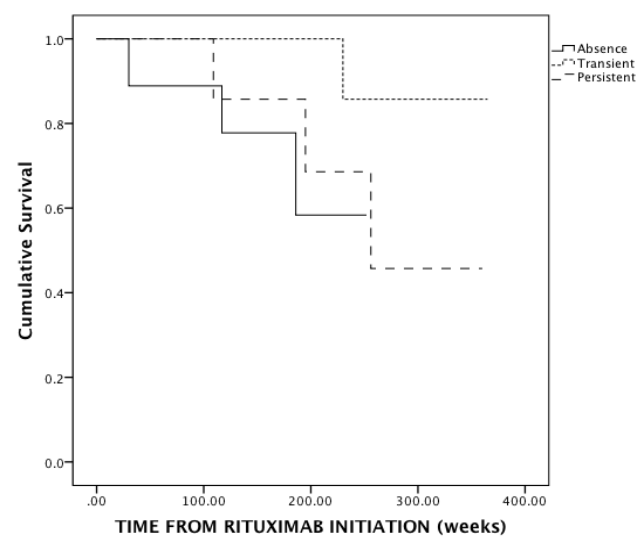

\begin{tabular}{l|r|r|r|r} 
Week & 0 & 100 & 200 & 300 \\
\hline $\mathrm{N}$ & 29 & 27 & 15 & 4
\end{tabular}

D Log rank $p=0.122$

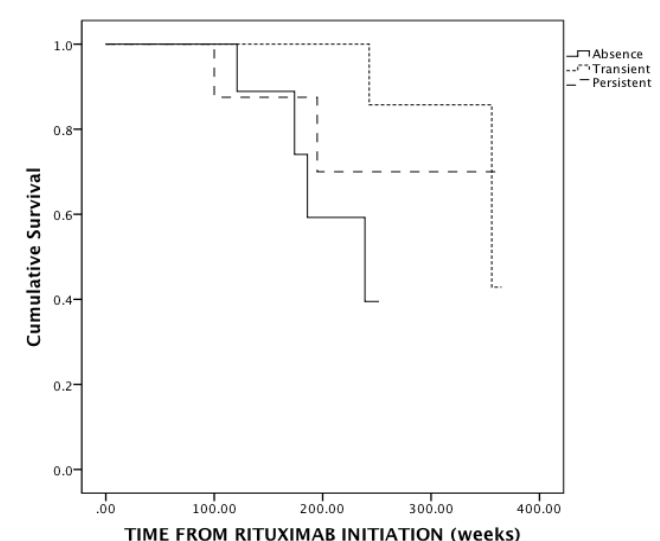

\begin{tabular}{l|r|r|r|r} 
Week & 0 & 100 & 200 & 300 \\
\hline $\mathrm{N}$ & 29 & 28 & 16 & 4
\end{tabular}


Figure 2 (on next page)

Kaplan-Meier analysis of the probability of relapse (A), severe (B) and chronic (C) infections and discontinuation due to hypogammaglobulinemia (D) according to change in Staphylococcus aureus nasal carriage status

Figure 2 
A Log Rank $p=0.756$

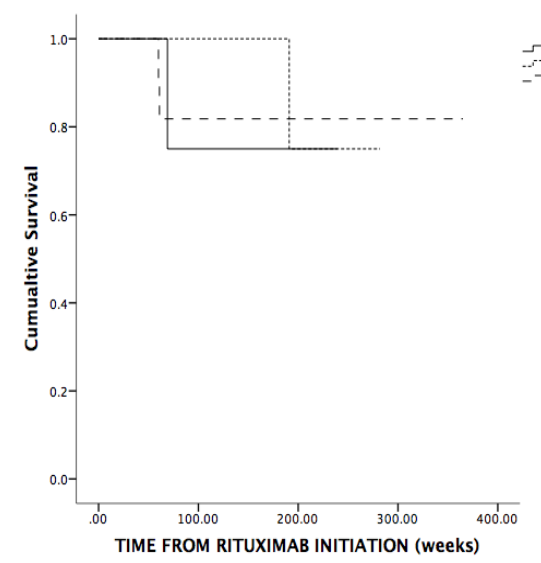

C Log Rank $p=0.121$

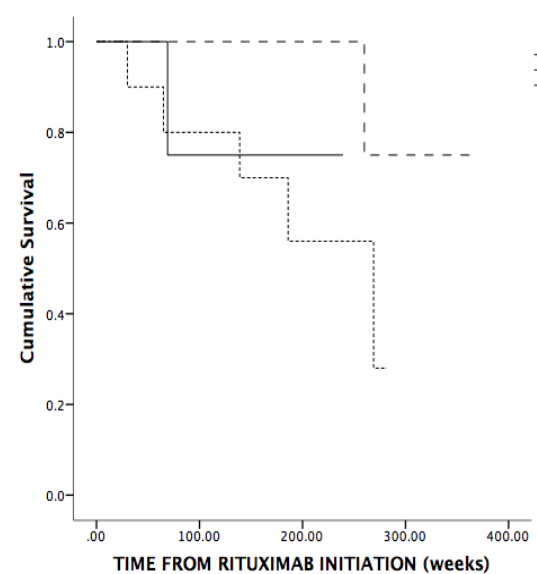

B Log Rank $p=0.913$

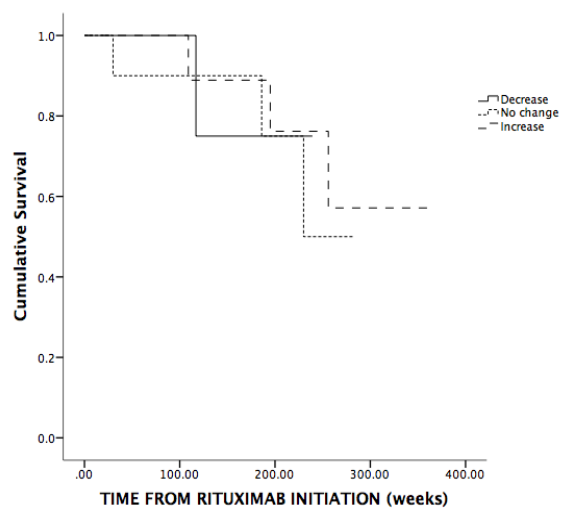

D Log Rank $p=0.474$

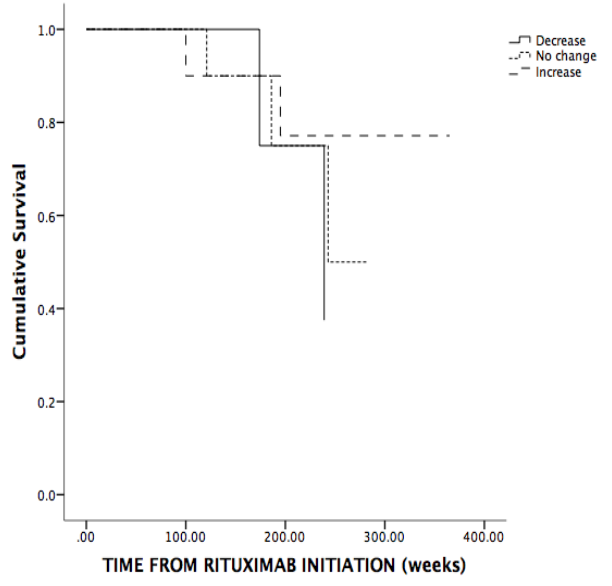


Figure 3 (on next page)

Kaplan-Meier analysis of the probability of hypogammaglobulinemia according to Trimethoprim-sulfamethoxazole (TMP-SMX) (A) and ciprofloxacin (B) antibiotic prophylaxis during rituximab maintenance

Figure 3 


\section{A Log rank $p=0.046$}

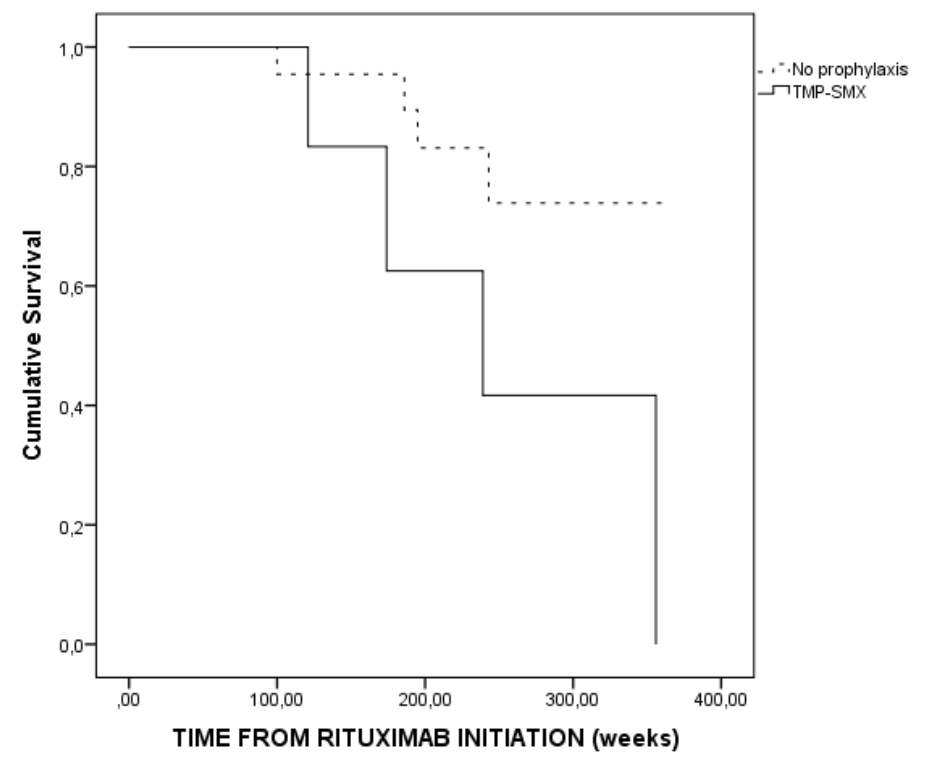

B Log rank $p=0.065$

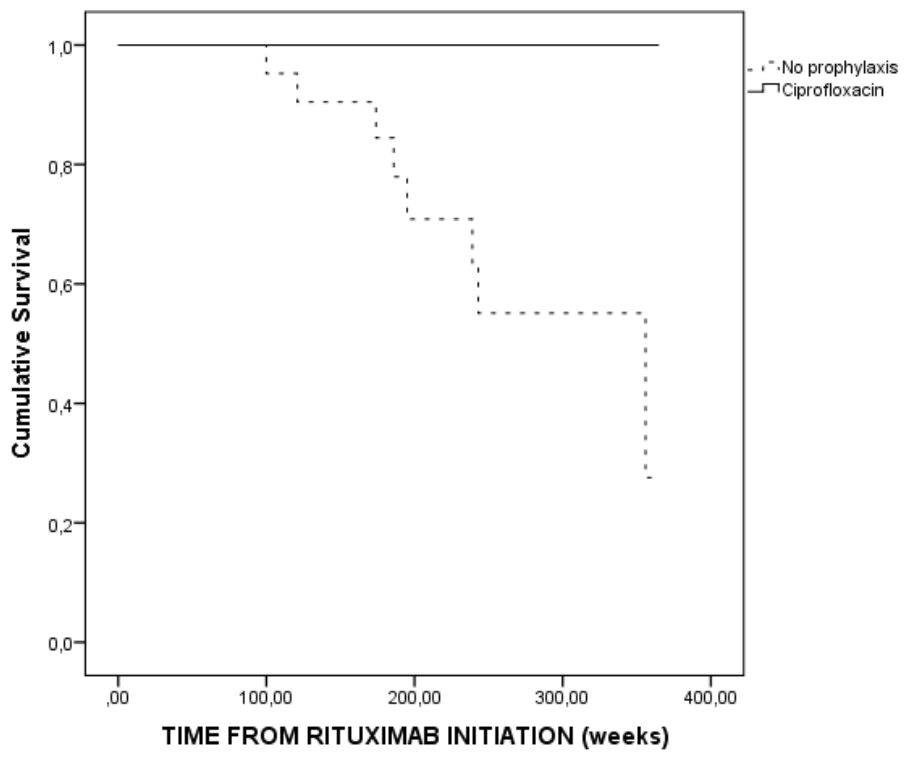

